\title{
Presencia de Aedes (Stegomyia) aegypti (Linnaeus, 1762) y su infección natural con el virus del dengue en alturas no registradas para Colombia
}

\author{
Freddy Ruiz-López¹, Ana González-Mazo¹, Andrés Vélez-Mira1, Giovan F. Gómez¹, \\ Luisa Zuleta ${ }^{1,2}$, Sandra Uribe ${ }^{1,2}$, Iván Darío Vélez-Bernal ${ }^{1}$ \\ 1 Programa de Estudio y Control de Enfermedades Tropicales, PECET, Universidad de Antioquia, Medellín, \\ Colombia \\ 2 Grupo de Investigación en Sistemática Molecular, Universidad Nacional de Colombia, Medellín, Colombia
}

Introducción. Aedes aegypti es el principal vector de fiebre amarilla urbana, dengue, chikungunya y zika. Se ha demostrado que la distribución biogeográfica de esta especie se ha expandido debido al calentamiento global y a factores socioeconómicos y culturales. Los cambios en los patrones de la distribución altitudinal de este vector y su infección con el virus son prioridades de la investigación encaminada a desarrollar estrategias de vigilancia entomológica y virológica en salud pública.

Objetivo. Evaluar la presencia de $A$. aegypti y su infección natural por el virus del dengue en alturas superiores a los 1.800 msnm en dos municipios periféricos del Valle de Aburrá, Antioquia, Colombia.

Materiales y métodos. Se instalaron 21 ovitrampas en los municipios de Bello y San Pedro de los Milagros, en un rango altitudinal de 1.882 a $2.659 \mathrm{msnm}$. Los adultos que emergieron de las ovitrampas se evaluaron con reacción en cadena de la polimerasa en tiempo real (RT-PCR) para la detección del virus del dengue.

Resultados. Se recolectaron 367 adultos de $A$. aegypti, siete de los cuales se encontraron a una altitud de 2.302 msnm en Tierradentro, Bello. Se detectaron 12 especímenes de $A$. aegypti positivos para dengue serotipo 2 en el barrio París de Bello, a $1.984 \mathrm{msnm}$.

Conclusión. Por primera vez se registró $A$. aegypti a 2.302 msnm, la mayor altitud registrada para este vector en Colombia. De igual forma, se encontró infección con el virus del dengue a 1.984 msnm. Estos hallazgos son significativos, ya que determinan regiones de Colombia con riesgo potencial de transmisión autóctona de dengue y otros arbovirus por $A$. aegypti.

Palabras clave: Aedes aegypti, altitud, ubicaciones geográficas, dengue, Colombia. doi: http://dx.doi.org/10.7705/biomedica.v36i2.3301

Presence of Aedes (Stegomyia) aegypti (Linnaeus, 1762) and its natural infection with dengue virus at unrecorded heights in Colombia

Introduction: Aedes aegypti is the main vector of urban yellow fever, dengue, chikungunya and Zika viruses. The biogeographical distribution of this species has expanded due to global warming, and socioeconomic and cultural factors. The changes in the altitudinal distribution patterns of this vector and its natural infection are priority fields of research to develop entomological, virological and public health surveillance strategies.

Objective: To evaluate the presence of $A$. aegypti and its natural infection with dengue virus in altitudes above 1.800 meters above sea level in two peripheral municipalities of the Valle de Aburrá, Antioquia, Colombia.

Materials and methods: Twenty-one ovitraps were set in the municipalities of Bello and San Pedro de los Milagros, at altitudes ranging from 1.882 to 2.659 masl. Emerged adults caught in the ovitraps were tested by RT-PCR for dengue virus detection.

Results: We collected $367 \mathrm{~A}$. aegypti adults, seven of which were found as high as 2.302 masl in Tierradentro, Bello. We detected serotype 2 dengue infection in 12 A. aegypti specimens collected in the neighbourhood of París, in Bello, at 1.984 masl.

\section{Contribución de los autores:}

Freddy Ruiz-López: identificación del material entomológico

Ana González-Mazo, Andrés Vélez-Mira, Luisa Zuleta, Freddy Ruiz-López: salida de campo y revisión de las ovitrampas Luisa Zuleta: procesamiento del material entomológico

Giovan F. Gómez y Sandra Uribe: recolección, determinación taxonómica y determinación de infección por el virus del dengue Iván Darío Vélez: diseño de la investigación, financiación y elaboración del manuscrito

Todos los autores participaron en el diseño de la investigación y en la escritura del manuscrito. 
Conclusion: We recorded $A$. aegypti at 2.302 masl, so far the highest altitudinal record in Colombia for this vector. Furthermore, mosquitoes collected at 1.984 masl were positive for dengue virus. These findings are significant as they identify regions in Colombia at risk of potential autochthonous transmission of dengue and other arboviruses by $A$. aegypti.

Key words: Aedes aegypti, altitude, geographic locations, dengue, Colombia. doi: http://dx.doi.org/10.7705/biomedica.v36i2.3301

Aedes (Stegomyia) aegypti es conocido como el principal vector de fiebre amarilla urbana (1), dengue (2), chikungunya (3) y zika (4). Es originario de África y se cree que la forma ancestral era un mosquito zoofílico que se criaba en los huecos de los árboles $(5,6)$. Poblaciones ancestrales, como la subespecie $A$. aegypti formosus, todavía se encuentran en África (7). Según Petersen (8) y Tabachnich (6), las poblaciones que habitaban en el bosque se concentraron en el norte de África durante un largo periodo de sequía ocurrido en el Sahara hace 4.000 millones de años aproximadamente. Es probable que estas condiciones extremas favorecieran su adaptación a las condiciones domésticas y dieran lugar a su reproducción en criaderos artificiales, en particular, en recipientes para el almacenamiento de agua $(6,8)$. Con el inicio del comercio mundial hace cientos de años, esta especie se desplazó a través de las zonas tropicales y subtropicales hasta llegar al Nuevo Mundo en los barcos del comercio de esclavos entre los siglos XV y XVIII (6). Después de su establecimiento en las zonas costeras de las regiones tropicales y templadas de las Américas, se dispersó hacia el interior del continente (9).

Debido al calentamiento global y a múltiples factores socioeconómicos regionales y locales, los patrones de distribución de $A$. aegypti han cambiado, con nuevos registros altitudinales y una extensa distribución en todos los continentes, incluidos Norteamérica, Europa y Asia $(10,11)$. En la ciudad de Puebla (México), esta especie pasó de 1.630 msnm en 1987 (12) a 2.133 msnm en el 2011 (13). En Venezuela se encuentra por debajo de los $2.000 \mathrm{msnm}$ (14). En Colombia, se pensaba que la especie no habitaba por encima de los $1.585 \mathrm{msnm}$ antes del trabajo de Suárez y Nelson en 1981 (15); sin embargo, estos autores lo registraron en 22 municipios por encima de los $1.600 \mathrm{msnm}$ y a 2.200

Correspondencia:

John Freddy Ruiz-López, PECET, Universidad de Antioquia, Calle $62 \mathrm{~N}^{\circ}$ 52-59, laboratorio 632, Medellín, Colombia

Teléfono: (574) 219 6502, fax: (574) 2196511

fredyruiz9@gmail.com

Recibido: 01/04/16; aceptado: 26/04/16 msnm en Málaga (Santander), siendo este último el registro altitudinal más alto para Suramérica hasta ahora. Según el Grupo de Entomología de la Dirección de Redes en Salud Pública del Instituto Nacional de Salud de Colombia, A. aegypti se encuentra en 718 municipios distribuidos en todos los departamentos del territorio nacional a altitudes que no sobrepasan los $2.200 \mathrm{msnm}$.

El conocimiento sobre los cambios en los patrones de distribución de $A$. aegypti y la comprensión de la forma en que sobrevive en límites superiores de altura, constituye una prioridad a nivel mundial y un reto para entender la biología y la dinámica de este vector en esas condiciones ambientales.

El objetivo de esta investigación fue evaluar la presencia de $A$. aegypti en límites de distribución altitudinal no reportados para Colombia, en particular por encima de los $1.800 \mathrm{msnm}$, en un transecto altitudinal entre los municipios de Bello y San Pedro de los Milagros, Antioquia, así como determinar el estatus de la infección natural por el virus del dengue.

\section{Materiales y métodos}

Debido a la situación actual en torno a los arbovirus (dengue, chikungunya y zika) en Colombia, y con base en los estudios adelantados por el Programa de Estudio y Control de Enfermedades Tropicales, PECET de la Universidad de Antioquia sobre el virus del dengue en el municipio de Bello, se utilizó una metodología rápida de muestreo para evaluar la presencia de $A$. aegypti en alturas superiores a los $1.800 \mathrm{msnm}$ en la periferia del Valle de Aburra.

Asimismo, se evaluó la infección por el virus del dengue en la mayoría de los mosquitos recolectados. Se colocaron 21 ovitrampas dentro y en los alrededores de las viviendas (corredores exteriores) en un rango altitudinal entre los 1.882 y los $2.659 \mathrm{msnm}$ (cuadro 1). Las ovitrampas se revisaron a los ocho días y los huevos recolectados se transportaron al insectario del PECET, donde posteriormente fueron inundados y criados hasta alcanzar el estado adulto bajo condiciones controladas de humedad $(70 \%)$ y temperatura $\left(26^{\circ} \mathrm{C}\right)$. 
Cuadro 1. Ubicación de las ovitrampas

\begin{tabular}{|c|c|c|c|c|}
\hline Ovitrampa & Municipio & Localidad & Altura (msnm) & Coordenada \\
\hline $\mathrm{T} 1$ & Bello & Jalisco & 2.237 & N $6.31422^{\circ} \mathrm{W} 75.59703^{\circ}$ \\
\hline $\mathrm{T} 2$ & Bello & San Félix & 2.390 & N $6.33059^{\circ} \mathrm{W} 75.59734^{\circ}$ \\
\hline T3 & Bello & San Félix & 2.512 & N $6.34089^{\circ} \mathrm{W} 75.60789^{\circ}$ \\
\hline $\mathrm{T} 4$ & Bello & La Unión & 2.584 & N $6.43070^{\circ} \mathrm{W} 75.59208^{\circ}$ \\
\hline T5 & Bello & El Tambo & 2.556 & N $6.43069^{\circ} \mathrm{W} 75.59208^{\circ}$ \\
\hline T6 & San Pedro de los Milagros & Casco urbano & 2.489 & $\mathrm{~N} 6.45888^{\circ} \mathrm{W} 75.55675^{\circ}$ \\
\hline T7 & San Pedro de los Milagros & Cerezales & 2.659 & N $6.41673^{\circ} \mathrm{W} 75.56425^{\circ}$ \\
\hline T8 & San Pedro de los Milagros & Cerezales & 2.592 & N $6.41784^{\circ} \mathrm{W} 75.55998^{\circ}$ \\
\hline T9 & Bello & Tierradentro* & 2.302 & N $6.37931^{\circ} \mathrm{W} 75.59347^{\circ}$ \\
\hline $\mathrm{T} 10$ & Bello & Altos de Niquía & 2.252 & $\mathrm{~N} 6.37934^{\circ} \mathrm{W} 75.59348^{\circ}$ \\
\hline $\mathrm{T} 11$ & Bello & París & 1.968 & N $6.18877^{\circ} \mathrm{W} 75.37169^{\circ}$ \\
\hline $\mathrm{T} 12$ & Bello & París & 1.943 & $\mathrm{~N} 6.18880^{\circ} \mathrm{W} 75.35124^{\circ}$ \\
\hline $\mathrm{T} 13$ & Bello & París** & 1.984 & $\mathrm{~N} 6.18904^{\circ} \mathrm{W} 75.37278^{\circ}$ \\
\hline $\mathrm{T} 14$ & Bello & París & 1.957 & $\mathrm{~N} 6.18104^{\circ} \mathrm{W} 75.35290^{\circ}$ \\
\hline $\mathrm{T} 15$ & Bello & Nueva Jerusalén & 1.977 & N $6.19241^{\circ} \mathrm{W} 75.35224^{\circ}$ \\
\hline $\mathrm{T} 16$ & Bello & París & 1.907 & N $6.18849^{\circ} \mathrm{W} 75.35038^{\circ}$ \\
\hline $\mathrm{T} 17$ & Bello & París & 1.883 & N $6.18930^{\circ} \mathrm{W} 75.35031^{\circ}$ \\
\hline $\mathrm{T} 18$ & Bello & París & 1.891 & N $6.18883^{\circ} \mathrm{W} 75.35033^{\circ}$ \\
\hline $\mathrm{T} 19$ & Bello & París & 1.882 & N $6.18822^{\circ} \mathrm{W} 75.35023^{\circ}$ \\
\hline $\mathrm{T} 20$ & Bello & París & 1.882 & $\mathrm{~N} 6.18645^{\circ} \mathrm{W} 75.35053^{\circ}$ \\
\hline $\mathrm{T} 21$ & Bello & Nueva Jerusalén & 1.977 & N $6.19241^{\circ} \mathrm{W} 75.35024^{\circ}$ \\
\hline
\end{tabular}

* Trampa ubicada a la mayor altura donde se recolectó Aedes aegypti

** Aedes aegypti positivos para el virus del dengue serotipo 2

Después de que los adultos emergieron, su identidad taxonómica se verificó usando las claves para mosquitos de importancia médica de la Walter Reed Biosystematics Unit (WRBU, http:// www.wrbu.org/command_aors_MQkeys.html). Los especímenes obtenidos de trampas entre los 1.882 y los $1.984 \mathrm{msnm}$ se usaron para formar grupos de tres a seis individuos, los cuales se procesaron para la detección viral usando la metodología de Kuno (16). Los demás mosquitos se montaron en alfileres entomológicos siguiendo los protocolos del Museo de Historia Natural de Londres (17).

\section{Resultados}

En las 11 ovitrampas instaladas en Bello entre los 1.882 y los $1.984 \mathrm{msnm}$, se recolectaron 360 adultos de $A$. aegypti. De los adultos obtenidos, se seleccionaron 40 individuos representativos de todas las trampas para formar grupos y hacer la detección viral. En dos grupos (12 individuos) de la trampa T13, ubicada a $1.984 \mathrm{msnm}$, se detectó la presencia del virus del dengue serotipo 2 (cuadro 1). Este serotipo se ha detectado y aislado previamente mediante métodos serológicos y moleculares en pacientes que asistían a consulta en la Clínica de Fiebre del barrio París (no se presentan los datos).

En las diez ovitrampas instaladas entre los 2.237 y $2.659 \mathrm{msnm}$, se observó ovipostura de $A$. aegypti en dos de ellas: la ovitrampa número T9 y la T10, ubicadas en Bello a 2.302 msnm (vereda Tierradentro, zona rural) y en Altos de Niquía, zona semiurbana, a $2.252 \mathrm{msnm}$, respectivamente. Después de la inundación de los huevos, en la ovitrampa 9 se observaron siete larvas, las cuales se criaron hasta la fase adulta y se obtuvieron cuatro hembras y tres machos, todos identificados como A. aegypti (figura 1). En la ovitrampa 10 los huevos no fueron viables y no se observó eclosión.

\section{Discusión}

Desde que Suárez, et al., incrementaron el rango de distribución de $A$. aegypti en Colombia de 1.600 a 2.200 msnm en 1981 (15), no se conocían estudios que evaluaran la distribución altitudinal de este importante vector en el país. En México, Lozano-Fuentes, et al. (13), reportaron A. aegypti a una altura máxima de $2.130 \mathrm{msnm}$, y encontraron altas densidades por debajo de los $1.700 \mathrm{msnm}$, en tanto que su presencia no fue tan frecuente entre los 1.700 y los 2.130 msnm, incluida la ciudad de Puebla, la cual se encuentra a $2.100 \mathrm{msnm}$. En Venezuela Navarro, et al. (14), realizaron un estudio de los géneros y especies de mosquitos registrados por encima de los $2.000 \mathrm{msnm}$ y conservados en museos y colecciones, con el fin de conocer las de mayor distribución altitudinal, y no encontraron $A$. aegypti en ninguno de los 9.607 registros de 432 localidades revisados. Según estos autores (14), solo se conocen registros de 

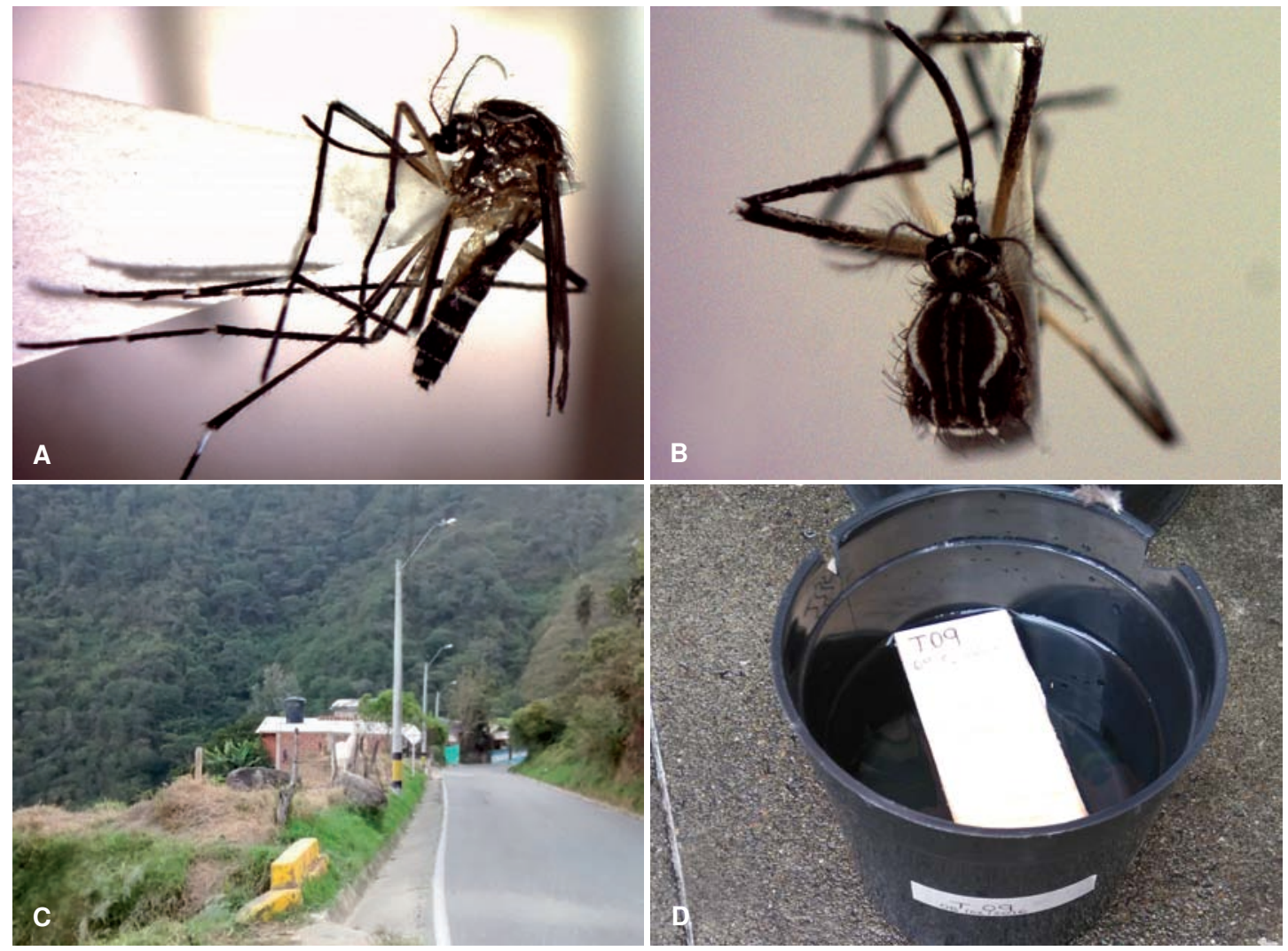

Figura 1. Aedes aegypti adulto y sus caracteres diagnósticos (líneas blancas en forma de lira en el tórax), obtenido de la ovitrampa T9 a 2.302 msnm, en la vereda Tierradentro, municipio de Bello, Antioquia. A. Vista lateral. B. Vista dorsal del tórax. C. Vivienda donde fue ubicada la ovitrampa. D. Ovitrampa: recipiente con agua y una tablilla de balso en su interior.

A. aegypti en Venezuela por debajo de los 2.000 msnm, lo que podría ser una barrera altitudinal para la transmisión del dengue y otros arbovirus transmitidos por este vector.

En Colombia, De la Hoz, et al. (18), reportaron casos de dengue asociados a la presencia de $A$. aegypti de altura en Cáqueza, Cundinamarca, a 1.746 msnm. Según Padilla, et al. (19), entre 1999 y 2010, en el Sistema Nacional de Vigilancia en Salud Pública, Sivigila, se registraron 628.016 casos de dengue, de los cuales 2.801 casos se presentaron en 53 municipios ubicados por encima de los $1.800 \mathrm{msnm}$ (19). También en el estado de Guerrero (México), Herrera-Basto confirmó la transmisión autóctona de dengue a $1.700 \mathrm{msnm}$ (20), lo cual generó la alerta sobre la distribución del vector en zonas de mayor altitud. Recientemente, en marzo de 2016, el director departamental de salud de la Paz, Bolivia (SEDES), confirmó la presencia de $A$. aegypti a $2.600 \mathrm{msnm}$ en las ciudades de Cochabamba y Tarija; sin embargo, no se ha evaluado la transmisión de dengue en estas altitudes (no se publican los datos).
Es de anotar que este es un estudio piloto que actualmente se lleva a cabo en Bello y San Pedro de los Milagros en el marco de la respuesta a la transmisión del dengue, de tanta importancia para el país, y al incremento en la transmisión de otros arbovirus como el del chikungunya y del zika, cuyo vector es también $A$. aegypti. En este sentido, los autores consideran que en el futuro se deben realizar estudios entomológicos, con estimativos de las densidades y periodos más largos de vigilancia entomológica, para descartar que la presencia de A. aegypti en las zonas más altas, donde el número de huevos fue bajo, no haya sido una infestación accidental, ya que las ovitrampas se colocaron durante ocho días únicamente.

La presencia de $A$. aegypti en nuevas altitudes y áreas rurales puede estar relacionada con los asentamientos humanos en las laderas de la montaña y a los lados de las nuevas vías de acceso intermunicipales y departamentales, lo cual no significa desestimar el papel del calentamiento global y su impacto en todos los ecosistemas, incluido el incremento en el rango de distribución 
de insectos vectores $(21,22)$. Infortunadamente, el cambio climático y su relación con la transmisión de arbovirus en Colombia se han estudiado poco (23-25).

Aunque las densidades de $A$. aegypti no son tan altas por encima de los $2.200 \mathrm{msnm}(11,13,15)$, su presencia en áreas rurales por encima de dicha altitud llama a contemplar la ampliación del rango altitudinal de intervención en las campañas de control vectorial. Para ello sería importante considerar, además de las densidades del vector, la presencia de dengue y de los serotipos virales que circulan simultáneamente en los pacientes y el vector.

Los resultados del presente estudio podrían considerarse como una alerta sobre la presencia del vector en límites superiores a los conocidos y deberían evaluarse en términos del riesgo potencial de transmisión autóctona de arbovirus trasmitidos por este mosquito en áreas que se consideraban a salvo del riesgo de transmisión debido a que su altitud y temperatura actúan como barreras.

Sería pertinente realizar actividades de vigilancia entomológica en zonas de altura en el Valle de Aburra, particularmente en el municipio de Bello, dado el desplazamiento y el asentamiento de comunidades hacia las comunas y veredas ubicadas en las laderas y partes altas de la montaña. Estos hallazgos constituyen un llamado de atención en términos de los cambios en los patrones de distribución de este vector en Colombia y en relación con la situación actual y futura de la vigilancia y el control vectorial.

\section{Conflicto de intereses}

Los autores declaran no tener conflicto de intereses.

\section{Financiación}

Esta investigación fue financiada por el Programa de Estudio y Control de Enfermedades Tropicales, PECET, de la Universidad de Antioquia, el Proyecto "Eliminar el dengue, un desafío para Colombia" y la Universidad Nacional de Colombia, sede Medellín.

\section{Agradecimientos}

A Evencio Mosquera por el trabajo en el insectario y con las oviposturas del límite superior de altura. A todo el equipo de entomología de campo y de insectario del proyecto "Eliminar el dengue, un desafío para Colombia" (Jovany Barajas, Sandra Ángel, Sandra Gómez, Sebastián Durán, Luz Adriana Muñoz, Sebastián Pérez). A Johan
Acevedo y David Gallo, del Grupo de Investigación en Sistemática Molecular de la Universidad Nacional de Colombia, por su colaboración en el procesamiento de material para los estudios biológicos y moleculares.

\section{Referencias}

1. Monath TP. Yellow fever. The arboviruses: Epidemiology and ecology. In: Monath TP, editor. Boca Ratón (FL): CRC Press; 1989. p. 139-231.

2. Simmons CP, Farrar JJ, Chau NV, Wills B. Dengue. N Engl J Med. 2012;366:1423-32. http://dx.doi.org/10.1056/ nejmra1110265

3. Pialoux G, Gauzere BA, Jaureguiberry S, Strobel M. Chikungunya, an epidemic arbovirosis. Lancet Infect Dis. 2007;5:319-27. http://dx.doi.org/10.1016/s1473-3099(07) 70107-x

4. Marchette NJ, García R, Rudnick A. Isolation of Zika virus from Aedes aegypti mosquitoes in Malaysia. Am J Trop Med Hyg. 1969;18:411-5.

5. Mattingly PF. Genetical aspects of the Aedes aegypti problem I. Taxonomy and bionomics. Ann Trop Med Parasit. 1957;51:392-408.

6. Tabachnick WJ, Munstermann LE, Powell JR. Genetic distinctness of sympatric forms of Aedes aegypti in East Africa. Evolution. 1979;33:287-95. http://dx.doi.org/10. $2307 / 2407619$

7. Brown JE, Evans BR, Zheng W, Obas V, Barrera-Martínez L, Egizi A, et al. Human impacts have shaped historical and recent evolution in Aedes aegypti, the dengue and yellow fever mosquito. Evolution. 2014;68:514-25. http://dx.doi. org/10.1111/evo.12281

8. Petersen JL. Behavior differences in two subspecies of Aedes aegypti (L.) (Diptera: Culicidae) in East Africa (Dissertation). Notre Dame, IN: University of Notre Dame; 1977.

9. Lounibos LP. Invasions by insect vectors of human disease. Annu Rev Entomol. 2002;47:233-66. http://dx.doi. org/10.1146/annurev.ento.47.091201.145206

10. Kraemer MU, Sinka ME, Duda KA, Mylne QN, Shearer MF, Barker MC, et al. The global distribution of the arbovirus vectors Aedes aegypti and Ae. albopictus. Elife. 2015;4:e08347. http://dx.doi.org/10.7554/eLife.08347

11. Dhimal M, Gautam I, Joshi HD, O'Hara RB, Ahrens B, Kuch $\mathrm{U}$. Risk factors for the presence of chikungunya and dengue vectors (Aedes aegypti and Aedes albopictus), their altitudinal distribution and climatic determinants of their abundance in Central Nepal. PLoS Negl Trop Dis. 2015:9;e0003545. http:// doi.org/10.1371/journal.pntd.0003545

12. Ibáñez-Bernal S. Nuevo registro altitudinal de Aedes (Stegomyia) aegypti (Linnaeus, 1762) (Diptera: Culicidae) en México. Folia Entomol Mex. 1987;72:163-164.

13. Lozano-Fuentes S, Hayden MH, Welsh-Rodríguez C, Ochoa-Martínez C, Tapia-Santos B, Kobylinski CK, et al. The dengue virus mosquito vector Aedes aegypti at high elevation in México. Am J Trop Med Hyg. 2012;87:902-9. http://dx.doi.org/10.4269/ajtmh.2012.12-0244 
14. Navarro JC, Ventura FD, Zorrilla A, Liria J. Registros de mayor altitud para mosquitos (Diptera: Culicidae) en Venezuela. Rev Biol Trop. 2010;58:245-54. http://dx.doi. org/10.15517/rbt.v58i1.5206

15. Suárez FM, Nelson JM. Registro de altitud del Aedes aegypti en Colombia. Biomédica. 1981;1:225. http://dx.doi. org/10.7705/biomedica.v1i4.1809

16. Kuno G. Universal diagnostic RT-PCR protocol for arboviruses. J Virol Methods. 1998;72:27-41. http://dx.doi. org/10.1016/s0166-0934(98)00003-2

17. Ruiz-López F. Molecular systematics of the Oswaldoi complex (Anopheles; Nyssorhynchus) in South America. Dissertation. London: University of Kent and The Natural History Museum; 2010.

18. Rodríguez H, De la $\mathrm{Hoz}$ F. Dengue and dengue and vector behaviour in Cáqueza, Colombia, 2004. Rev Salud Pública. 2005;7:1-15. http://dx.doi.org/10.1590/s012400642005000100001

19. Padilla JC, Rojas DP, Sáenz-Gómez R. Dengue en Colombia: epidemiología de la reemergencia a la hiperendemia. Bogotá: Guías de Impresión, Ltda.; 2012. p. 249.

20. Herrera-Basto E, Prevots DR, Zárate ML, Silva JL, Sepúlveda-Amor J. First reported outbreak of classical dengue fever at 1,700 meters above sea level in Guerrero State, México, June 1988. Am J Trop Med Hyg. 1992;46:649-53.

21. Hales S, Wet N, Maindonald J, Woodward A. Potential effect of population and climate changes on global distribution of dengue fever: An empirical model. Lancet. 2002;360:830-4. http://dx.doi.org/10.1016/S0140-6736(02)09964-6

22. Khormi HM, Kumar L. Climate change and the potential global distribution of Aedes aegypti: Spatial modelling using GIS and CLIMEX. Geospat Health. 2014;8:405-15. http:// dx.doi.org/10.4081/gh.2014.29

23. Cassab A, Morales V, Máttar S. Factores climáticos y casos de dengue en Montería, Colombia. 2003-2008. Rev Salud Pública. 2011;13:115-28. http://dx.doi.org/10.1590/ s0124-00642011000100010

24. Rúa-Uribe G, Calle D, Rojo R, Henao E, Sanabria W, Suárez C. Influencia del evento climático El Niño sobre la dinámica de transmisión de dengue en Medellín, AntioquiaColombia. Iatreia. 2012;25:314-22.

25. Rúa-Uribe GL, Suárez-Acosta C, Chauca J, Ventosilla $P$, Almanza R. Modelado del efecto de la variabilidad climática local sobre la transmisión de dengue en Medellín (Colombia) mediante análisis de series temporales. Biomédica. 2013; 142-52. http://dx.doi.org/10.7705/biomedica.v33i0.1444 